\title{
Glutamate hypothesis of depression and its consequences for antidepressant treatments
}
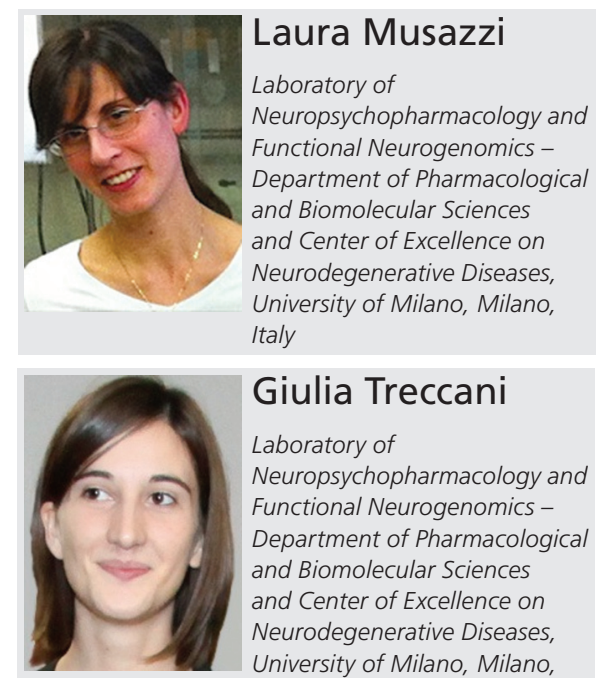

Giulia Treccani

Laboratory of

Neuropsychopharmacology and Functional Neurogenomics Department of Pharmacological and Biomolecular Sciences and Center of Excellence on Neurodegenerative Diseases, University of Milano, Milano, Italy

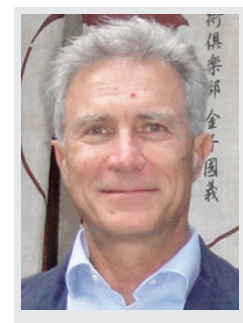

\section{Maurizio Popoli}

Author for correspondence: Laboratory of

Neuropsychopharmacology and Functional Neurogenomics Department of Pharmacological and Biomolecular Sciences and Center of Excellence on Neurodegenerative Diseases, University of Milano, Milano Italy

Tel.: + 390250318361

Fax: +390250318278

maurizio.popoli@unimi.it

\section{\begin{tabular}{l} 
EXPERT \\
REVIEWS \\
\hline
\end{tabular}}

\author{
"Recent growing evidence suggests that glutamatergic \\ neurotransmission, the major excitatory system in the brain, \\ plays a critical role in the pathophysiology and treatment of \\ neuropsychiatric disorders."
}

Depression is the fourth leading cause of disability and disease worldwide. WHO projections indicate that depression will be the highest ranked cause of disease burden in the middle- and high-income countries by the year $2030[1]$.

Drugs that increase the synaptic availability of monoamines, including tricyclic antidepressants, selective serotonin reuptake inhibitors, serotonin and norepinephrine reuptake inhibitors, and dopamine reuptake inhibitors, have been used to treat depression for more than 50 years. However, these drugs have significant limitations, including a long time lag for therapeutic response (weeks to months), low response rates and side effects serious enough to induce discontinuation in nearly $20 \%$ of patients. Moreover, the delayed response is suggestive of an involvement in antidepressant therapeutic action of slow-onset adaptations in downstream signaling pathways regulating neuroplasticity and cellular resilience $[2,3]$.

Recent growing evidence suggests that glutamatergic neurotransmission, the major excitatory system in the brain, plays a critical role in the pathophysiology and treatment of neuropsychiatric disorders. The glutamatergic system is interconnected with GABAergic and monoaminergic pathways, and it has been shown that approximately $80 \%$ of neurons in the neocortex are excitatory glutamatergic neurons [4]. In the last several years, a number of clinical and preclinical studies demonstrated that maladaptive changes in excitatory/inhibitory circuitry, particularly in glutamate homeostasis and neurotransmission, have a primary role in mood and anxiety disorders.

First, it has been shown that glutamate levels are increased in plasma and in postmortem samples of frontal cortex from patients with major depressive disorder, and antidepressants restore normal plasma glutamate levels $[5,6]$. Abnormalities in total brain levels of glutamate and its metabolite glutamine were also investigated in vivo using proton magnetic resonance spectroscopy. While results varied, a large majority of studies have provided evidence of reduced glutamate metabolite levels in the frontal cortex and cingulate regions of depressed patients during depressive episodes (reviewed in [7]). Interestingly, glutamate metabolite levels normalize during clinical remission, suggesting a pathophysiological importance during active episodes of major depression. Moreover, a recent work showed that the reduction of glutamate levels in the anterior cingulate cortex of depressed patients normalized with electroconvulsive therapy treatment and was associated with clinically assessed therapeutic response [8].

Moreover, several postmortem studies have shown altered mRNA and protein expression of glutamate receptors ( $N$-methylD-aspartate receptors, a-amino-3-hydroxy- 
5-methyl-4-isoxazolepropionic acid receptors and metabotropic glutamate receptors [mGluRs]) in select brain areas from depressed individuals, suggesting that dysregulation of glutamatergic receptor signaling is likely to be associated with the disorder [9].

\section{“...brain morphometric studies of patients with major depression have demonstrated volumetric reduction of prefrontal regions ... brain regions closely associated with stress response and emotional/cognitive processing."}

Furthermore, expression of the glial excitatory amino acid transporters, responsible for glial glutamate reuptake, was found to be reduced in individuals with major depression, suggesting reduced extracellular glutamate clearance [10]. Since glia participates in the uptake, metabolism and recycling of glutamate, it was hypothesized that a reduction of glial activity may account for the alterations in glutamate neurotransmission observed in depression. Interestingly, neuropathological studies in postmortem brains from patients with major depression reported consistent evidence for reduced glial cell number and/or density in the brain regions with glutamatergic predominance, including orbital, anterior cingulate, subgenual and dorsolateral prefrontal cortices [11]. Similarly, brain morphometric studies of patients with major depression have demonstrated volumetric reduction of prefrontal regions, especially the orbital frontal cortex, the anterior cingulate cortex and the subgenual cingulate cortex, and of hippocampus, brain regions closely associated with stress response and emotional/cognitive processing [12]. Interestingly, volumetric reductions and the decrease of glial cell number and density observed in depressed subjects are localized in the same brain areas, suggesting a possible correlation between these phenomena.

This hypothesis has also received support from numerous preclinical studies reporting brain structural remodeling in animal models of depression. Animal models are widely used to study the pathophysiology of mood disorders. Since behavioral stress is recognized as a main risk factor for mood and anxiety disorders, most animal models of depression are based on stress exposure. Subjecting experimental animals to behavioral stress is a classical way of inducing measurable neuroendocrine, behavioral, neurochemical and morphological changes, resembling those observed in depressed subjects. Animal studies suggest a role for glucocorticoid signaling, activated by stressors in both rodents and humans and altered in depressed individuals, and the consequent enhancement of glutamate release in the morphological/ functional changes induced by stress. Indeed, a number of studies reported a rapid, transient and marked increase of extracellular glutamate in different areas, including the hippocampus, amygdala and prefrontal cortex of rats subjected to different stressors or to treatment with corticosterone (the main stress hormone in rodents; reviewed in $[4,13])$. It was also demonstrated, using different methodologies, that the rapid increase of extracellular glutamate levels is related to increased depolarization-evoked release, mediated by corticosterone membrane-located receptors (mineralocorticoid receptor in hippocampus, glucocorticoid in prefrontal and frontal cortex) coupled to nongenomic (protein synthesis-independent) mechanisms [14,15]. Chronic antidepressants nearly or completely abolish the stress-induced upregulation of glutamate release in cortical areas, suggesting that this drug effect may be a relevant component of the therapeutic action of antidepressants.

Moreover, it was shown that chronic exposure to behavioral stress induces atrophy, retraction and simplification of dendritic arbor in both hippocampal CA3 area and medial prefrontal cortex, changes that are reversible both after the cessation of stress and antidepressant treatment $[4,16]$.

The simplification of dendritic arbor induced in the hippocampus and medial prefrontal cortex of stressed rats is considered, together with the loss of glial cells, one of the likely mechanisms accounting for the volumetric changes registered in the hippocampus and prefrontal cortex areas of humans with major depression. Indeed, while changes in the size and shape of dendritic shafts and spine density are typical physiological mechanisms of activity-dependent synaptic plasticity, higher and sustained extracellular concentrations of glutamate may cause dendrite retraction and impair glial and neuronal function.

All these preclinical and clinical lines of evidence have provided the background for the formulation of a new hypothesis for the etiopathogenesis of depression: the glutamate hypothesis of depression [4,17]. In this conceptual framework, a sustained glutamate accumulation, caused by alterations of glutamate release, clearance and metabolism, in selected brain areas associated with cognitive-emotional behaviors and mood regulation, is recognized as the cause of the structural/functional changes and impaired synaptic activity in these regions.

66...simplification of dendritic arbor ... is considered, together with loss of glial cells, one of the likely mechanisms accounting for the volumetric changes

registered in hippocampus and prefrontal cortex areas of humans with major depression."

Further support for this hypothesis comes from a number of clinical studies showing rapid and prolonged antidepressant action of glutamate receptor antagonists, particularly ketamine. Ketamine, a noncompetitive $N$-methyl-D-aspartate receptor antagonist, is used as a standard anesthetic agent at doses as high as $2 \mathrm{mg} / \mathrm{kg}$. Intriguingly, different studies have reported a robust, rapid (within $2 \mathrm{~h}$ ) and sustained ( $\sim 1$ week) antidepressant effect in treatment-resistant patients with major depressive disorder of a single infusion of ketamine at subanesthetic doses $(0.5 \mathrm{mg} / \mathrm{kg}$ $[18,19])$. However, limitations in the clinical use of ketamine due to its poor safety profile (psychotomimetic effects, cognitive impairments, tolerance) impede a widespread application of this molecule in the treatment of depression. For this reason, a number of drugs affecting glutamatergic neurotransmission (selective NR2B antagonists, positive modulators of a-amino3-hydroxy-5-methyl-4-isoxazolepropionic acid receptor and mGluR ligands) are in development as potential antidepressant agents. In particular, accumulating recent preclinical evidence 
has shown a consistent antidepressant effect of group II mGluR (mGluR2/3) ligands (administered in combination with existing antidepressants) and Group III mGluR selective agonists (mainly of mGluR7 and mGluR8) or allosteric modulators (of mGluR4 [20]).

Although early-phase clinical trials of drugs are now in progress, larger randomized clinical trials are needed to establish the potential therapeutic efficacy of these drugs, based on the glutamate hypothesis of depression.

"...a sustained glutamate accumulation, caused by alterations of glutamate release, clearance and metabolism, in selected brain areas associated with cognitive-emotional behaviors and mood regulation, is recognized as the cause of the structural/ functional changes and impaired synaptic activity..."

Moreover, the dampening action of chronic antidepressants on the stress-induced upregulation of glutamate release, observed in preclinical models [15], suggests a new putative line of drug development directly targeted at the molecular mechanisms regulating glutamate release $[4,13]$. In this context, a pharmacological modulation of presynaptic release of glutamate might provide a means of attenuating exaggerated or inadequate stress response in mood/anxiety disorders. On the other hand, since several antidepressants tested, endowed with distinct primary mechanisms, are all able to abolish the effects of stress on glutamate release [15], we hypothesize that this could be a selective property of antidepressants related with their therapeutic action. For this reason, we propose the measurement of the preventive effect of drugs on stress-induced glutamate release as a functional test to assay molecules with antidepressant/anxiolytic action.

There are several challenges that remain to be addressed by novel antidepressant therapies. Ideal new drugs should obtain a rapid and sustained remission of symptoms and have minimal cognitive side effects. The rapid action of glutamate receptor antagonists is certainly a fascinating and promising feature for third-generation antidepressants, allowing for immediate clinical effects with possibly higher rates of remission.

\section{Financial \& competing interests disclosure}

$M$ Popoli has received research support andlor has been a consultant for Abiogen, GlaxoSmithKline, Merck Sharp \& Dohme, Abbott, Servier and Fidia. The authors have no other relevant affiliations or financial involvement with any organization or entity with a financial interest in or financial conflict with the subject matter or materials discussed in the manuscript apart from those disclosed.

No writing assistance was utilized in the production of this manuscript.

\section{References}

1 Mathers CD, Loncar D. Projections of global mortality and burden of disease from 2002 to 2030. PLoS Med. 3(11), e442 (2006).

2 Krishnan V, Nestler EJ. The molecular neurobiology of depression. Nature 455(7215), 894-902 (2008).

3 Racagni G, Popoli M. The pharmacological properties of antidepressants. Int. Clin. Psychopharmacol. 25(3), 117-131 (2010).

4 Sanacora G, Treccani G, Popoli M. Towards a glutamate hypothesis of depression: an emerging frontier of neuropsychopharmacology for mood disorders. Neuropharmacology 62(1), 63-77 (2012).

5 Hashimoto K, Sawa A, Iyo M. Increased levels of glutamate in brains from patients with mood disorders. Biol. Psychiatry 62(11), 1310-1316 (2007).

6 Küçükibrahimoglu E, Saygin MZ, Caliskan M, Kaplan OK, Unsal C, Gören MZ. The change in plasma GABA, glutamine and glutamate levels in fluoxetine- or $S$-citalopramtreated female patients with major depression. Eur. J. Clin. Pharmacol. 65(6), 571-577 (2009).
7 Yüksel C, Öngür D. Magnetic resonance spectroscopy studies of glutamate-related abnormalities in mood disorders. Biol. Psychiatry 68(9), 785-794 (2010).

8 Zhang J, Narr KL, Woods RP, Phillips OR, Alger JR, Espinoza RT. Glutamate normalization with ECT treatment response in major depression. Mol. Psychiatry doi:10.1038/mp.2012.46 (2012) (Epub ahead of print).

9 Beneyto M, Kristiansen LV, Oni-Orisan A, McCullumsmith RE, Meador-Woodruff $\mathrm{JH}$. Abnormal glutamate receptor expression in the medial temporal lobe in schizophrenia and mood disorders. Neuropsychopharmacology 32(9), 1888-1902 (2007).

10 Choudary PV, Molnar M, Evans SJ et al. Altered cortical glutamatergic and GABAergic signal transmission with glial involvement in depression. Proc. Natl Acad. Sci. USA 102(43), 15653-15658 (2005).

11 Rajkowska G, Miguel-Hidalgo JJ. Gliogenesis and glial pathology in depression. CNS Neurol. Disord. Drug Targets 6(3), 219-233 (2007).

12 Koolschijn PC, van Haren NE, LensveltMulders GJ, Hulshoff Pol HE, Kahn RS. Brain volume abnormalities in major depressive disorder: a meta-analysis of magnetic resonance imaging studies. Hum. Brain Mapp. 30(11), 3719-3735 (2009).

13 Popoli M, Yan Z, McEwen BS, Sanacora G. The stressed synapse: the impact of stress and glucocorticoids on glutamate transmission. Nat. Rev. Neurosci. 13(1), 22-37 (2012).

14 Olijslagers JE, de Kloet ER, Elgersma Y, van Woerden GM, Joëls M, Karst $\mathrm{H}$. Rapid changes in hippocampal CA1 pyramidal cell function via pre- as well as postsynaptic membrane mineralocorticoid receptors. Eur. J. Neurosci. 27(10), 2542-2550 (2008).

15 Musazzi L, Milanese M, Farisello P et al. Acute stress increases depolarization-evoked glutamate release in the rat prefrontal/ frontal cortex: the dampening action of antidepressants. PLoS ONE 5(1), e8566 (2010).

16 Pittenger C, Duman RS. Stress, depression, and neuroplasticity: a convergence of mechanisms. Neuropsychopharmacology 33(1), 88-109 (2008).

17 McCarthy DJ, Alexander R, Smith MA et al. Glutamate-based depression GBD. Med. Hypotheses 78(5), 675-681 (2012). 
18 Zarate CA Jr, Singh JB, Carlson PJ et al. A randomized trial of an $N$-methylD-aspartate antagonist in treatmentresistant major depression. Arch. Gen. Psychiatry 63(8), 856-864 (2006).
19 Valentine GW, Mason GF, Gomez R et al. The antidepressant effect of ketamine is not associated with changes in occipital amino acid neurotransmitter content as measured by [(1)H]-MRS. Psychiatry Res. 191(2), 122-127 (2011).
20 Krystal JH, Mathew SJ, D’Souza DC, Garakani A, Gunduz-Bruce H, Charney DS. Potential psychiatric applications of metabotropic glutamate receptor agonists and antagonists. CNS Drugs 24(8), 669-693 (2010). 